\title{
Tangence
}

\section{Changement et auto-théorisation}

\section{Un point de vue psychanalytique sur quelques enjeux de \\ l'autobiographie chez Sartre}

\section{Jean-François Chiantaretto}

Numéro 42, décembre 1993

Le récit de soi

URI : https://id.erudit.org/iderudit/025790ar

DOI : https://doi.org/10.7202/025790ar

Aller au sommaire du numéro

Éditeur(s)

Tangence

ISSN

0226-9554 (imprimé)

1710-0305 (numérique)

Découvrir la revue

Citer cet article

Chiantaretto, J.-F. (1993). Changement et auto-théorisation : un point de vue psychanalytique sur quelques enjeux de l'autobiographie chez Sartre.

Tangence, (42), 80-99. https://doi.org/10.7202/025790ar d'utilisation que vous pouvez consulter en ligne.

https://apropos.erudit.org/fr/usagers/politique-dutilisation/ 


\section{Changement et auto-théorisation. Un point de vue psychanalytique sur quelques enjeux de l'autobiographie chez Sartre}

Jean-François Chiantaretto *

Lorsque le lecteur parvient à la deux cent huitième des deux cent quatorze pages des Mots ${ }^{1}$, une surprise l'attend. L'auteur y présente en ces termes cette autobiographie en forme de récit d'enfance:

Voilà mon commencement: je fuyais, des forces extérieures ont modelé ma fuite et m'on fait. À travers une conception périmée de la culture, la religion transparaissait, qui servit de maquette [...]

J'ai changé. Je raconterai plus tard [...] par quelle raison je fus amené à penser systématiquement contre moi-même [...] L'illusion rétrospective est en miettes [...]

J'ai désinvesti mais je n'ai pas défroqué: j'écris toujours. Que faire d'autre? [...]

Du reste, ce vieux bâtiment ruineux, mon imposture, c'est aussi mon caractère: on se défait d'une névrose, on ne se guérit pas de soi. ${ }^{2}$

Voila mon commencement... j'ai changé: le renvoi final au bors-texte d'un changement ayant déjà eu lieu, qui vaut comme la suite dans la vie de l'enfance narrée dans le texte, consacre la disparition comme tel du présent de l'écriture, soit sa domination totale. Le point de vue de l'écrivain autobiographe de 1963 ne fait mine de s'énoncer in fine que pour mieux se masquer au lecteur, derrière la confusion instaurée entre deux futurs: le futur hors texte de l'enfant des Mots (le changement ayant eu lieu), le futur

\footnotetext{
* Psychanalyste, chargé de cours à L'Université Paris 7. Membre de la rédaction de la revue Le Coq-Héron. Anime un groupe de recherches, "Littérature personnelle et psychanalyse". A publié Bertolt Brecht penseur intervenant, Paris, Éd. Publisud, 1985. À paraître fin 1994: Psychanalyse et écriture: la question de l'autobiograpbie.

1 Jean-Paul, Sartre, Les mots, coll. "Folio", Paris, Gallimard, 1964.

2 Ibid., p. 208-213.
} 
d'une écriture à venir de la suite de l'enfance. Ainsi se perpétue par et pour Les mots ce point de vue du futur qui n'y serait énoncé que pour être dénoncé.

C'est toute la question du changement - altération/altérité - , qui est posée dans et par cet ultime changement de perspective des Mots. Elle travaille d'une manière ou d'une autre toute écriture de soi et l'autobiographie stricto sensu se spécifierait de la refouler comme telle tout en la mettant à plat dans l'écriture: en la dissolvant dans le cours, linéaire ou fragmenté, du tracé de la vie. Sartre va toutefois au plus loin dans ce sens en dramatisant la mise hors texte du changement avec ce dispositif final du "Voilà mon commencement [...] j'ai changé". Pour interpréter correctement celle-ci, c'est-à-dire pour ne pas manquer sa fonction intratextuelle en la prenant trop à la lettre, la lecture des Mots avec ses précédents non fictionnels paraît fort utile.

Cela me permettra de mieux dégager le processus d'autothéorisation structurant le geste autobiographique sartrien. Ledit processus sera étudié ici en ce qu'il reprend certains axes théoriques de L'être et le néant portant notamment sur la conception du temps, de la mort et de la possession. L'idée avancée par moi de l'autobiographie comme biographie de soi mettant en acte un fantasme d'auto-engendrement trouvera ainsi à se préciser ${ }^{3}$.

\section{Écriture de soi et changement}

Comme l'ont bien relevé Philippe Lejeune et Serge Doubrovsky dans leur lecture des Carnets ${ }^{4}$, l'écriture de soi chez

3 J'ai développé cette idée pour Sartre dans Psychanalyse et écriture: la question de l'autobiographie. Ladite idée est au coeur de la théorisation psychanalytique de l'autobiographie que je propose dans cet ouvrage. J'en ai également avancé différents étayages, notamment dans: "Pour une approche psychanalytique de l'autobiographique. Prolégomènes", Psycbanalyse à l'Université, vol. XV, n 60,1990, p. 67-91; "De la biographie à l'autobiographie: la psychanalyse et l'autobiographique", Le Coq-Héron, no 118, 1990, p. 54-67; "Freud auto-biographe ou l'autobiographie par excès ", Le Coq-Héron, $n^{\circ} 126,1992$; "Fondation et auto-biographie après Freud. L'exemple de Maud Mannoni ", Psychanalyse à l'Université, vol. XVIII, $\mathrm{n}^{\circ} 71,1993$, p. 77-86.

4 Serge Doubrovsky, "Sartre: retouches à un autoportrait", dans Autobiographiques, Paris, P.U.F., coll. "Perspectives critiques", 1988, p. 123-167. Philippe Lejeune, "Les enfances de Sartre", Moi aussi, Paris, Seuil, coll, "Poétique", p. 117-163. 
82

Sartre correspond aux moments de rupture dans sa vie et son œuvre, à des moments de désolidarisation d'avec soi, sur le modèle de la mue du serpent précisément utilisé par lui-même le 19 décembre 1939, dans ses Camets ${ }^{5}$.

Toutefois, si l'image de Sartre vaut assurément pour tous les moments d'écriture de soi, au moins des Carnets aux Mots, la notion de changement est représentée dans l'écriture de façon sensiblement divergente dans ces deux textes. Dans les deux cas, le "tressage du vécu et du théorisé " 6 , pour reprendre l'expression de Doubrovsky, non seulement est patent mais encore est déterminé par le rapport à L'être et le néant. Ceci posé, même si le découpage chronologique y est largement biaisé par la structuration théorique, il reste que l'écriture descriptive du vécu de 1939-40, de par la forme journal mais pas seulement, donne lieu à un texte ouvert, alors que Les mots constitue un texte totalement fermé. Il en va ainsi parce que les Carnets ont fonction d'instituer l'exploration de l'espace intérieur comme une démarche d'expérimentation, de mise au point (au sens également des ultimes réglages) et de vérification de la théorie, du fait même que l'écriture est le lieu d'un changement, pour cet écrivain aux prises avec le réel (l'histoire).

Dans cette perspective, c'est tout le rapport de l'écriture de soi au temps qui s'avère différent, des Carnets aux Mots. Dans les Camets, le diariste accepte l'inachèvement de l'écriture de soi par soi-même, dans la mesure où celle-ci vient témoigner d'un travail de deuil à l'endroit d'une coïncidence de soi avec soi-même. L'écriture a fonction d'attester le changement dans la vie de l'auteur-narrateur ou, en termes plus sartriens, d'inscrire une désolidarisation de soi (passé). Sartre entend ainsi opposer ses Carmets à ceux de Gide.

[...] j'ai fait un retour sur mon carnet à moi et j'ai vu combien il différait de ceux de Gide. c'est d'abord un carnet de témoin. [...] Et moi aussi j'écris n'importe quoi sur mon carnet, mais c'est avec l'impression que la valeur historique de mon témoignage me justifie à le faire. [...] D'un autre point de vue et dans un esprit tout différent, ce journal est une remise en question de moi-même. [...] Ce sont des enregistrements et, en les écrivant, j'ai l'impression - fallacieuse - de laisser derrière

5 Cf. Les carnets de la drôle de guerre, Paris, Gallimard, 1983, p. 175.

6 Serge Doubrovsky, op. cit., p. 127. 
moi ce que j'écris. [...] J'essaye de constituer en écrivant une base solide et cristallisée d'où partir. En somme, il y a chez les primitifs des cérémonies pour aider le vivant à mourir, pour aider l'âme à se dégager du corps. Mes notes "confessionnelles" ont le même but: aider mon être présent à couler dans le passé, l'enfoncer un peu, au besoin. Il y a là une part d'illusion, car il ne suffit pas de dénoncer une constante psychologique pour la modifier. Mais au moins cela dessine-t-il des lignes de changement possible. ${ }^{7}$

C'est mon orgueil qui me fait parler, mon sens du progrès et cette façon que j'ai de me désolidariser de ce que j'étais la veille. Chaque fois que quelqu'un semble frappé par la permanence de mon moi, je suis égaré d'inquiétude. ${ }^{8}$

Il ne s'agit pas simplement, comme avec tout journal, d'inscrire en creux la place du futur dans l'enregistrement ou le recueillement des traces laissées par le temps qui passe. Il s'agit plus fondamentalement, dans la consécration même d'un je veux changer transmué en un je suis changé (par l'expérience de la guerre), d'inscrire l'à venir en adoptant le point de vue du futur. Le je suis changé, étayé sur un témoignage prétendument historique masquant/avouant sa valeur d'auto-témoignage, permet dans les Carnets de surmonter l'impossibilité d'occuper un point de vue sur la vie pendant la vie 9 ; non pas en instituant une relation isomorphique entre inachèvement de la vie et inachèvement de son écriture, mais en affirmant une homogénéité entre l'écriture et le changement, entre l'écriture du changement et le changement dans l'écriture, entre l'attestation d'une désolidarisation d'avec soi-même et l'affirmation de sa vie comme vécue du point de vue d'un futur de grand homme - de sa vie et non de sa vie écrite ${ }^{10}$.

Tel est bien l'endroit où l'opposition journal/autobiographie s'avère notablement insuffisante pour rendre ce qui différencie les Carnets et Les mots. Car les Carnets, à la différence des Lettres ${ }^{11}$ et comme le texte autobiographique, décrivent la vie en tant qu'elle a été vécue du point de vue du futur. On retrouve dans

\footnotetext{
7 1983, op. cit., p. $90-92$.

$8 \quad$ Ibid., p. 19

$9 \quad$ Ibid., p. 99.

10 Cf. ibid., p. 97.

11 On chercherait en vain dans les Lettres le point de vue du futur, à quelque niveau que ce soit.
} 
Les mots ce vécu de futur grand homme et les nombreux passages en forme de récits rétrospectifs, émaillant les Carmets, se lisent comme autant de préfigurations du texte autobiographique. Plus même, la lecture de certains de ces fragments d'écriture rétrospective donne parfois l'étrange impression ${ }^{12}$ de remonter au point où le texte autobiographique prétend arrêter sa reconstruction chronologique, à savoir ce que Philippe Lejeune a pu désigner comme "la coupure de $1916{ }^{13}$; coupure marquée par la coïncidence de la pré-puberté, du remariage de la mère et de la perte de la foi ${ }^{14}$. Il est d'ailleurs notable à cet égard que la sexualité soit aussi peu présente dans les deux cas. Je soulignerai surtout, pour le moment, l'homogénéité non moins étrange des deux textes quant à la pseudo-dénonciation de l'illusion biographique. Ici comme là ${ }^{15}$, malgré l'écart de quelques vingt-quatre années, s'affirme le geste comme permanent de décrire la permanence d'une vie vécue - dans l'illusion, maintenant dénoncée en tant qu'ordonnée par le futur et conséquemment, valant en elle-même pour un texte biographique - dont par définition la fin est connue et ordonne le tout.

Derrière la (relative) communauté de point de vue des deux textes pour décrire la vie telle qu'elle est ou a été vécue, Les mots se spécifient d'ordonner l'écriture elle-même du point de vue d'un futur changement - situé après la coupure de 1916, soit après les douze ans de l'auteur-narrateur, censés constituer la limite chronologique du récit d'enfance, malgré de nombreux dépassements -; changement renvoyé donc au hors-texte dans le passage final cité plus haut. Autrement dit, alors que le changement et l'inachèvement sont au coeur de l'écriture dans les Carnets, l'écriture dans Les mots a fonction d'unification rétrospective.

Pour analyser plus avant les enjeux tout à fait centraux de cette unification, il n'est pas inutile au passage de relever la cohérence stratégique de la "ruse" déployée par Sartre, quant à cette

12 Sans doute renforcée par ceci que nous lisons les Carnets après Les mots.

13 Cf. Philippe Lejeune, "L'ordre du récit dans Les mots de Sartre", dans Le pacte autobiographique, Paris, Seuil, coll. "Poétique", 1975, p. 197-243.

14 Je pense tout spécialement à ce passage, commençant ainsi: "J'ai perdu la foi à douze ans. Mais j'imagine que je n'ai jamais cru bien fort [...]" (1983, op. cit., p. 92-94).

$15 C f$. en particulier ibid., p. 103-106; pour Les mots, je renvoie au passage central concernant la transmission par le grand-père de "l'illusion rétrospective" (op. cit., p. 168-169). 
problématique du changement, dans l'écriture intime de cette période de guerre, contemporaine de l'écriture de L'être et le néant. De l'altération à l'altérité, le diariste (les Carnets) et l'épistolier (les Lettres) se complètent pour mieux réduire le "témoignage historique à une modalité d'écriture de soi, l'altération du monde (la guerre) à l'altération de soi et à l'opérateur d'un changement interne, l'adresse à l'autre (Simone de Beauvoir comme interlocuteur et destinataire externes) pour la mise au point du travail philosophique à un dialogue interne au sein duquel ledit autre n'est plus qu'un double ${ }^{16}$. Et la désolidarisation de soi affirmée dans le dialogue de (l'écriture de) la vie et de (l'écriture de) la philosophie s'avère en vérité biaisée par l'auto-prescription de l'énonciation philosophique, comme antidépresseur ayant fonction de maîtriser ou d'annuler l'affect dépressif lié à l'écart entre soi et soi-même avivé par la guerre.

[...] la philosophie que je fais [...] a un rôle dans ma vie qui est de me protéger contre les mélancolies, morosités et tristesses de la guerre et puis à cette heure je n'essaye pas de protéger ma vie après coup par ma philosophie, ce qui est salaud, ni de conformer ma vie à ma philosophie ce qui est pédantesque mais vraiment vie et philo ne font qu'un.

[...] il y aura ça dans ma vie, que j’aurai aimé une personne de toutes mes forces, sans passionnel et sans merveilleux mais du dedans. Mais il fallait que ce fût vous, mon amour, quelqu'un qui soit si étroitement mêlé à moi qu'on ne reconnaît plus le sien du mien. Je vous aime. ${ }^{17}$

Je lui [à Simone de Beauvoir] ai exposé les grandes thèses de L'être et le néant qui n'était pas encore écrit. C'était pendant la "drôle de guerre". Je lui ai exposé toutes mes idées quand elles étaient en voie de formation. [...] elle était la seule qui était à mon niveau de connaissance de moi, de ce que je voulais faire. Donc, elle était l'interlocuteur parfait, l'interlocuteur qu'on a jamais eu. [... $]^{18}$

16 Outre les citations ci après, les notations abondent dans les Lettres qui mettent en scène différentes modalités de réduction de Simone de Beauvoir, pour ainsi dire, à une non-autre. Par ailleurs, il faudrait analyser les écritures intimes de l'un et de l'autre comme une manière d'écriture intime double: une écriture de la complétude narcissique.

17 Lettres au Castor et à quelques autres, I (1926-1939), Paris, Gallimard, 1983, p. 39,184 .

18 "Entretiens sur moi-même ", dans Situations, X, Paris, Gallimard, 1976, p. 190. 
86

[...] Maintenant, je n'y crois pas à la littérature spontanée, mais à cette époque-là, j'y croyais. Mes lettres ont été en somme l'équivalent d'un témoignage sur ma vie.

S. de B. - Oui, mais pour porter ce témoignage il vous fallait un interlocuteur.

J.-P. S. - Oui. ${ }^{19}$

De l'utilisation de l'autre comme support et opérateur d'un écart entre soi et soi-même au dit écart vécu et conçu comme un arrachement de soi à l'autre, le lecteur - analyste ou simplement informé de psychanalyse - serait tenté d'établir un lien, à l'cndroit désigné par la coupure de 1916 comme le point aveugle du discours autobiographique: la coïncidence de la perte de la foi et du remariage de la mère. Il est très frappant de constater combien cette coïncidence est mise en absence dans les suites ou reprises autobiographiques postérieures aux Mots, alors même que les deux événements sont donnés pour tels. Ladite mise en absence est d'autant plus notable que l'opération est menée au plus près; ainsi du trouble de datation saisissant Sartre, qui à quelques phrases d'intervalle - précisément dans le dialogue avec son non-autre Simone de Beauvoir - situe alternativement la perte de la foi vers "l'âge de douze ans, à La Rochelle" et à "onze ans ", soit respectivement après et avant les débuts de la vie commune avec son beau-père - tout en affirmant que la question de Dieu ne se posera "jamais plus" 20 .

Cette question "jamais plus posée" réunit bien ce qui sera séparé dans le discours autobiographique: la "décision" de ne plus croire en Dieu et l'arrachement à la mère par cet intrus ${ }^{21}$ venant occuper la place du père mort. Sans doute est-il à cet endroit particulièrement tentant de céder à une lecture de type

19 "Entretiens avec Jean-Paul Sartre", dans Simone de Beauvoir, La cérémonie des adieux, Paris, Gallimard, 1981, p. 229-230.

20 Cf ibid., p. 545-546.

21 De la première (Jean sans terre) à la seconde version des Mots, l'intrusion a précisểment été effacée, comme si elle était incompatible avec l'affirmation pleine du geste autobiographique pour Sartre. 
psychobiographique, d'autant que le recours à la psychanalyse y a, pour ainsi dire, une place toute trouvée ${ }^{22}$. Dans cette perspective, la perte de la foi serait à mettre en correspondance avec l'attaque du registre de la toute-puissance des pensées - voire avec la perte de la croyance en ladite toute-puissance - et la séparation d'avec la mère, opérées par un tiers (en l'occurrence persécuteur). À partir de là, pourrait voguer la galère interprétative - de l'auteur en personne, entendu strictement comme la personne de l'auteur - qui aboutirait à définir la sexualité de Sartre en termes de fixation au stade phallique; fixation dont viendrait précisément témoigner dans Les mots l'arrêt du récit d'enfance avant la puberté et l'absence de la sexualité. Telle ne sera pas ma démarche, qui reste centrée sur la reconstruction autobiographique.

Je soulignerai plutôt que la coïncidence évoquée ci-dessus constitue comme le secret exhibé/caché par les suites des Mots, un secret désignant donc après coup le point aveugle du texte autobiographique de 1964. Point aveugle: l'expression est délibérément choisie pour ce qu'elle évoque le registre de l'originaire. Car nous allons voir en quoi l'effacement de la perte de la mère rejoint l'affectation de Dieu dans et par l'écriture. Le point aveugle constitue en fait le lieu même de l'acte autobiographique en tant qu'actualisation d'un fantasme d'auto-engendrement.

Les mots ont ceci de commun avec les Carnets que la sexualité y est comme telle absente. $\grave{A}$ y regarder de plus près, on constate également que la prégnance du registre religieux est très sensiblement plus marquée dans Les mots, essentiellement pour énoncer et dénoncer la valeur religieuse de l'écriture. Il en va là de la différence princeps séparant les deux textes, au delà de celles précédemment signalées. Dans les Carnets, je l'ai dit, l'écriture de soi participe de la mise au point de L'être et le néant; dans Les mots, la théorisation de Dieu - en tant qu'elle ordonne au sein du livre de 1943 une conception du temps et de la mort - fonctionne après coup comme la théorie de l'autobiographie qui y est mise en pratique.

22 Je pense bien évidemment au paradigme rendu manifeste avec la fameuse déclaration du narrateur des Mots, concernant son absence de surmoi. Déclaration qui pourrait s'interpréter comme la trace symptomatique d'un vécu persécutif. 


\section{Auto-théorisation, autobiographie et sexualisation de la pensée}

Philippe Lejeune a bien montré que l'autobiographie se veut chez Sartre la reconstruction de son projet et combien cette ligne d'écriture suppose, outre la notion même de projet, la théorisation du temps exposée dans L'être et le néant, en particulier quant à la critique de l'idée d'un passé considéré en soi ${ }^{23}$. Citons le passage suivant de ce livre, particulièrement significatif à cet égard:

[...] C'est que la seule force du passé lui vient du futur: de quelque manière que je vive ou que j'apprécie mon passé, je ne puis le faire qu'à la lumière d'un pro-jet de moi sur le futur. Ainsi l'ordre de mes choix d'avenir va déterminer un ordre de mon passé et cet ordre n'aura rien de chronologique. [...] Le projet actuel décide donc si une période définie du passé est en continuité avec le présent ou si elle est un fragment discontinu d'où l'on émerge et qui s'éloigne.

[...] ce projet progressif entraîne une série de décollements par rapport à mon passé. Le passé, c'est alors ce que je regarde du haut de mes progrès, avec une sorte de pitié un peu méprisante, c'est ce qui est strictement objet passif d'appréciation morale et de jugement $[\ldots]^{24}$

Dans le second fragment cité, on retrouve au plan théorique cette idée du changement telle qu'elle a donné lieu dans les Carnets à l'image de la mue du serpent et telle qu'elle correspond dans Les mots au renvoi hors texte à un changement ayant eu lieu dans l'après du récit d'enfance. On la retrouve toutefois associée à une définition du passé qui vaudra dans Les mots à deux niveaux: comme modèle des récits que se racontait l'enfant autour de la séduction imaginaire de la mère - "la suite au prochain numéro "25 - et comme dans la pseudo-dénonciation de l'«illusion rétrospective" gouvernant les biographies, qui vaut en fait pour l'exact énoncé de la démarche autobiographique des Mots: "[...] dans une vie terminée, c'est la fin qu'on tient pour la vérité du commencement" ${ }^{26}$. Il faut encore ajouter, à propos de

23 Cf. Philippe Lejeune, "L'ordre du récit dans Les mots de Sartre", op. cit. Comme l'auteur le souligne lui-même, cela constitue d'ailleurs le propre de toute autobiographie.

24 (1943), L'être et le néant, Paris, Gallimard, coll. "Tel", 1976, p. 556-558, 560.

25 (1964), op. cit., p 99-100.

26 Ibid., p. 169. 
ce passage, que non seulement il semble résonner dans le texte de 1964 avec le recours à l'idée de hauteur pour qualifier le point de vue du futur - soit le temps présent de l'écriture ${ }^{27}$ - mais surtout que la définition du passé fait fond sur une occupation de la place du père. Ainsi peut-on lire les premières lignes de "Mon passé ", dans lesquelles le premier exemple choisi constitue une évocation du père (inavouée, mais incontournable) comme la théorie par anticipation de cette place d'un absent telle qu'elle sera mise en jeu en 1964 - c'est-à-dire de la mise en acte d'écriture d'un fantasme.

Il n'en demeure pas moins que la liberté qui s'échappe vers le futur ne saurait se donner de passé au gré de ses caprices ni, à plus forte raison, se produire elle-même sans passé [...] le passé est ce qui est hors d'atteinte et qui nous hante à distance. S'il ne détermine pas nos actions, au moins est-il tel que nous ne pouvons prendre de décision nouvelle sinon à partir de lui. Si j'ai préparé l'École navale et si je suis devenu officier de marine, à quelque moment que je me reprenne et que je me considère, je suis engagé; [...] Je puis bien subitement m'insurger contre ce fait, donner ma démission, décider mon suicide [...] Mais c'est, au fond, reconnaitre son importance immense de plate-forme et de point de vue; toute action destinée à m'arracher à mon passé doit d'abord être conçue à partir de ce passé-là, c'est-à-dire doit avant tout reconnaître qu'elle naît à partir de ce passé singulier qu'elle veut détruire $[\ldots]^{28}$

Sartre dit je à la place de son père et propose ici une manière de synthèse des quelques traces biographiques qu'il possédait de lui. Le théoricien de "Mon passé" nous livre une auto-théorisation en forme d'autobiographie de son père; une autobiographie qui poserait le passé de l'autobiographié (le père) - reconstruit comme pouvant aboutir à un "suicide", soit à la mort comme fuite impossible de son passé - en tant qu'il constitue le passé de l'autobiographe (le fils) à partir duquel celui-ci a à naître.

Cette discrète émergence d'une très christique confusion des places du père et du fils désigne avant l'heure le fantôme du père mort qui vient hanter l'auteur-narrateur des Mots et y délimiter un espace scripturaire (et donc scriptural) au sein duquel la mort de l'un (le père) vient se confondre avec la naissance de l'autre (le

27 Ibid., cf. le passage déjà mentionné p. 54.

28 (1943), op. cit., p. 553. 
fils). L'anticipation théorique en 1943 des Mots est d'autant plus à souligner en tant que théorisation du temps (passé) qu'elle opère aussi en tant que théorisation de la mort. C'est de fait cette dernière qui doit être lue comme une théorie (masquée comme telle) de l'écriture autobiographique à venir. Ladite théorisation se développe sur le fond d'une critique du Sein zum Tode ('’̂trepour-la mort) de Heidegger:

Il suffisait de considérer la mort comme terme ultime appartenant à la série. [...] il n'y a plus d'autre côté de la vie, et la mort est un phénomène humain, c'est le phénomène ultime de la vie, vie encore. [...] Mais la mort ainsi récupérée ne demeure pas simplement humaine, elle devient mienne; en s'intériorisant, elle s'individualise; ce n'est plus le grand inconnaissable qui limite l'humain mais c'est le phénomène de ma vie personnelle qui fait de cette vie une vie unique, c'est-à-dire une vie qui ne recommence pas, où l'on ne reprend jamais son coup. Par là je deviens responsable de ma mort comme de ma vie. [...] ${ }^{29}$

L'être et le néant procède à une disjonction en le sujet humain de l'être temporel et de l'être mortel. Si le passé tire son sens du présent, la mort non seulement ne constitue pas le sens de la vie mais en outre, en marquant l'irruption de ce qui n'appartient pas (plus) au registre des possibilités du sujet - soit littéralement l'irruption du non sens de l'extérieur de sa vie vient ôter toute signification à sa vie ${ }^{30}$.

Il est très important de noter que le raisonnement opérant en 1943 pose l'irruption de la mort en tant qu'elle signe à la fois la (toute-)puissance de Dieu et celle d'autrui. Cette convergence s'avère essentielle pour mon propos mais il faut ajouter tout de suite qu'elle ne donne pas lieu à une coïncidence. Sans entrer ici dans une discussion de la conception sartrienne de l'idée de Dieu $^{31}$, il faut souligner que celle-ci ne se situe pas sur le même plan que la réalité d'autrui. L'idée de Dieu intervient dans la pensée de Sartre au titre d'un idéal; idéal ayant la consistance d'un désir définissant l'homme ${ }^{32}$. Rétroactivement, du point de vue des Mots, ce "désir d'être Dieu" me paraît renvoyer au désir

29 Ibid., p. 590-592.

30 Cf. ibid., p. 596-597.

31 Discussion que je serais tenté quant à moi de mener notamment à partir de la notion, bien peu sartrienne à vrai dire, de transcendance vide. 
présidant à l'écriture autobiographique. Mon affirmation doit être précisée: à Dieu désignant dans L'être et le néant un point de vue impossible sur la totalité, le point de fuite minant toute totalisation et désignant en creux l'impossibilité de l'en-soi-pour-soi, correspond l'impossibilité dans le registre de la conscience individuelle, d'une totalisation de soi dans la description de sa vie. Il n'y a pas de point de vue sur sa vie qui soit extérieur à celle-ci, affirme Sartre en $1943^{33}$.

C'est précisément cette totalisation de soi du point de vue d'autrui, pour tout dire cette biographie de soi, réputée impossible dans le texte philosophique comme elle l'était (prétendument) déjà dans les Camets $^{34}$, qui va en toute duplicité être mise en œuvre en 1964. Cette duplicité se retrouve d'ailleurs dans une des suites des Mots, lorsqu'il s'agit précisément d'expliquer en quoi la méthode biographique appliquée à Flaubert ne pouvait valoir aussi pour une méthode autobiographique: les deux raisons principales en seraient l'absence d'empathie pour soi-même et l'impossibilité d'une totalisation de la vie d'un homme vivant ${ }^{35}$.

Ladite duplicité est structurelle. Dans sa théorisation de la mort, Sartre ne confond pas Dieu et autrui. La mort marque le point d'achoppement du "désir d'être Dieu ", de l'idéal d'une fondation et d'une totale possession de soi dans et par la conscience de soi; et ce point d'achoppement signifie la "chute" dans l'ordre instauré par le point de vue d'autrui, la "victoire" de celui-ci ${ }^{36}$. La visée inavouée/inavouable de l'autobiographe est ainsi toute trouvée. Elle réside en ce lieu où l'altération de soi par le discours sur soi est suspendue définitivement mais où il reste à annuler la victoire d'autrui.

[...] toute description de sa propre vie, lorsqu'elle est tentée par le pour-soi est projet de soi par delà cette vie et, comme le

\footnotetext{
33 Gf. ibid., p. 349.

34 Cf. (1983), op. cit., p. 103-106, cité supra.

35 Cf. (1971), op. cit., p. 103-105. Et pourtant, Les mots me semble à lire comme un acte d'adhésion à sa propre enfance et comme une totalisation de celle-ci soumettant la chronologie au point de vue du futur (le présent de l'écriture).

36 Cf. (1943), op. cit., p. 598, 602.
} 
projet altérant est, du même coup, aggloméré à la vie qu'il altère, c'est la propre vie de Pierre qui métamorphosait son sens en se temporalisant continûment. Or, à présent que sa vie est morte, seule la mémoire de l'Autre peut empêcher qu'elle se recroqueville dans sa plénitude en soi en coupant toutes ses amarres avec le présent.

[...] la vie décide de son propre sens, parce qu'elle est toujours en sursis, elle possède par essence un pouvoir d'auto-critique et d'auto-métamorphose qui fait qu'elle se définit comme un "pasencore" ou qu'elle est, si l'on veut, comme changement de ce qu'elle est. La vie morte ne cesse pas pour cela de changer et pourtant, elle est faite. [...] Il ne s'agit pas seulement pour elle d'une totalisation arbitraire et définitive; il s'agit, en outre, d'une transformation radicale: rien ne peut plus lui arriver de l'intérieur, elle est entièrement close, on ne peut plus rien y faire entrer; mais son sens ne cesse point d'être modifié du dehors. [...] la mort représente une totale dépossession [...] Ainsi l'existence même de la mort nous aliéne tout entier, dans notre propre vie, au profit d'autrui. Être mort, c'est être en proie aux vivants. ${ }^{37}$

La victoire sur la victoire d'autrui, sur les changements opérés post mortem du point de vue d'autrui, tiendra pour l'autobiographe à sa capacité à occuper dans l'écriture de sa vie le point de vue de l'autre, soit à écrire sa vie du point de vue de la (sa) mort. En d'autres termes, l'enjeu consiste à écrire sa vie de telle façon que l'écriture en constitue la version totalisée et définitive, c'est-à-dire définitivement hors d'atteinte de tout changement, de toute altération par soi-même et par autrui. Et de ce point de vue, pour ainsi dire, la mort et la naissance sont confondues dans une même dévalorisation, dans une même réduction à un fait.

C'est à cause de l'autre que ma mort est ma chute hors du monde, à titre de subjectivité au lieu d'être anéantissement de la conscience et du monde. Il y a donc un indéniable et fondamental caractère de fait, c'est-à-dire une contingence radicale dans la mort comme dans l'existence d'autrui. [...] Et méditer sur ma vie en la considérant à partir de la mort, ce serait méditer sur ma subjectivité en prenant sur elle le point de vue de l'autre; nous avons vu que cela n'est pas possible. [...] La mort est un pur fait, comme la naissance; elle vient à nous du dehors et elle nous transforme en dehors. Au fond, elle ne se

37 Ibid., p. 599, 601. 
distingue aucunement de la naissance, et c'est l'identité de la naissance et de la mort que nous nommons facticité. ${ }^{38}$

Voilà donc circonscrit pour l'essentiel ce que le philosophe Sartre réputait impossible en 1943 et ce que l'autobiographe Sartre va s'occuper à rendre possible, sans le dire ou, plus exactement, en le déniant. La (les) liaison (s) savamment élaborée (s), dans la totalisation de l'enfance proposée avec Les mots, de la naissance de l'un ('enfant-autobiographe) et de la mort de l'autre (le père) puis de sa réincarnation dans le grand-père, trouve sa modélisation théorique vingt ans plus tôt ${ }^{39}$.

$$
\text { *** }
$$

Ces passages de L'être et le néant rendent intelligible le rapprochement qui s'impose, à la lecture des Mots, de l'absence de justification (auto)biographique du prétendu arrêt à l'âge de douze ans, de l'absence de la sexualité comme telle, du caractère central de ce que j'appellerais le fantasme sororal (la mère comme sour incestueuse) et de la prégnance du registre religieux. Le divin camouflage de la sexualité révèle, pour ainsi dire, le fond de l'affaire, à savoir la problématique de la possession: à la fois comme possession de l'autre (aussi bien au sens courant de posséder quelqu'un) et comme possession par l'autre ${ }^{40}$.

Tout est clair des sources de sa névrose pour le narrateur des Mots. L'enfant sans père, pris dans la relation oedipienne mère/ grand-père, est fantasmatiquement identifié à un petit frère de sa mère et vit sa relation incestueuse avec elle sur ce mode. À la suite de quoi cet "inceste platonique" devient le modèle fantasmatique régissant la sexualité de l'adulte ou, en termes plus psychanalytiques - qui néanmoins correspondraient à l'autointerprétation que nous sert Sartre - , prend la force d'une fixation barrant l'accès à la sexualité génitale.

38 Ibid., p. 603-604.

39 Les conceptions sartriennes de l'Être-pour-autrui et du projet seraient à analyser plus globalement dans cette perspective.

40 Ici encore, il y aurait lieu d'étudier plus systématiquement lapproche de la conceptualisation sartrienne, cette fois de la possession telle qu'elle est développée en 1943. 
94

Karl [le grand-père] fredonnait parfois:

On n'peut pas êt' plus procb' parents

Que frère et scur assurément...

Ça me troublait: si l'on m'eût donné, par chance, une scur, m'ê̂t-elle été plus proche qu'Anne-Marie? Que Karlémami [les grand-parentsl? Alors c'eût été mon amante. Amante n'était encore qu'un mot ténébreux que je rencontrais souvent dans les tragédies de Corneille. Des amants s'embrassent et se promettent de dormir dans le même lit (étrange coutume: pourquoi pas dans des lits jumeaux comme nous faisions, ma mère et moi?). Je ne savais rien de plus mais sous la surface lumineuse de l'idée, je pressentais une masse velue. Frère, en tout cas, j'eusse été incestueux. J'y rêvais. Dérivation? Camouflage de sentiments interdits? C'est bien possible. J'avais une sceur aînée, ma mère, et je souhaitais une sour cadette. Aujourd'hui encore - 1963 - c'est bien le seul lien de parenté qui m'émeuve. J'ai commis la grave erreur de chercher souvent parmi les femmes cette sour qui n'avait pas eu lieu [...]

J'ai longtemps rêvé d'écrire un conte sur deux enfants perdus et discrètement incestueux. On trouverait dans mes écrits des traces de ce fantasme. [...] Ce qui me séduisait dans ce lien de famille, c'était moins la tentation amoureuse que l'interdiction de faire l'amour: feu et glace, délices et frustration mêlées, l'inceste me plaisait s'il restait platonique. ${ }^{41}$

Le lecteur, analyste ou simplement informé de psychanalyse, n'aurait plus alors qu'à prendre l'auteur au mot ${ }^{42}$ et pourrait s'autoriser à comprendre l'absence de toute sexualité manifeste dans le texte autobiographique comme la marque de l'effacement de la sexualisation de la pensée engagée par ce rapproché pathogène $^{43}$. Cette position de lecture, qui consiste à décrypter le textuel pour rendre compte du sexuel (des fantasmes sexuels de l'auteur en personne), peut s'avérer légitime ici, si le discours

41 (1964), op. cit., p. 48-49, note 1, p. $48-49$.

42 Le couple Sartre-Beauvoir pourrait d'ailleurs être interprété, dans la ligne de mon propos supra situant "Le Castor" en tant que double de l'auteur des Lettres, en termes de débouché comme naturel du modèle infantile du frère incestueux.

43 À cet égard, les rêveries à partir de la mère et les réitérations du geste consistant à différer la fin du récit ("la suite au prochain numéro", p. 99-100) pourraient également se lire comme la trace de scénarios masturbatoires refoulés. 
s'appuie sur une analyse globale et différentielle de l'ensemble des textes et principalement, en l'occurrence, des diverses versions fournies par Sartre de son infantile ${ }^{44}$, qu'elles soient fictionnelles, biographiques ou auto-référentielles. Ce ne saurait bien évidemment être ma démarche ici, d'autant plus qu'elle a déjà été menée à bien par Josette Pacaly, dans Sartre au miroir ${ }^{45}$. Cela lui permet - ce sera également le cas de Doubrovsky quelques années plus tard, dans une ligne de recherches plus localisée et assez différente ${ }^{46}$ - de bien mettre en relief, et de façon fondée, le nécessaire déplacement d'accent, par rapport à l'auto-interprétation de l'auteur-narrateur des Mots, vers l'identification maternelle. Un passage y invite tout spécialement:

Il regardait fixement ma mère, mais c'est à moi qu'il s'adressa: "On te gâte, petit, on te gâte! répétait-il avec précipitation. D'abord, je ne fis que m'offenser: on ne me tutoyait pas si vite; mais je surpris son regard maniaque et nous ne fîmes plus, Anne-Marie et moi, qu'une seule jeune fille effarouchée qui bondit en arrière. [...] j'ignorais tout de la chair et je n'imaginais pas ce que cet homme nous voulait mais l'évidence du désir est telle qu'il me semblait comprendre et que, d'une certaine manière, tout m'était dévoilé. Ce désir, je l'avais ressenti à travers Anne-Marie; à travers elle, j'avais appris à flairer le mâle, à le craindre, à le détester. Cet incident resserra nos liens $[\ldots]^{47}$

Sous l'identification à un frère incestueux, dont l'effacement serait en somme désigné comme tel par l'auteur-narrateur, se fait jour l'identification à la mère, objet d'un refoulement dont l'écriture autobiographique vient témoigner. La question n'est pas pour moi de reconstituer les fantasmes gouvernant la vie sexuelle de Sartre, même si, en dehors des traces textuelles, les déclarations de celuici y invitent plutôt massivement ${ }^{48}$; non pas par elles-mêmes l'entretien autobiographique ne présente pas plus de garantie intrinsèque quant à la "vérité " qu'un texte, autobiographique,

44 Au sens strictement freudien du fameux "l'inconscient, c'est l'infantile en nous".

45 Josette Pacaly, Sartre au miroir, Paris, Klincksieck, 1980. Je tiens à souligner ici la dette qui est la mienne vis-à-vis de ce livre remarquable, ouvrage de référence souvent utilisé et bien moins souvent cité... Ma position de lecture me semble converger avec la méthode suivie par cet auteur, en ce qu'elle n'évite pas l'auteur en personne.

46 Serge Doubrovsky, "Sartre: retouches à un autoportrait ", op. cit.

47 Ibid., p. 184.

48 Cf. notamment: (1975), op. cit., p. 116; (1974), op. cit., p. 385; ibid., p. 400. 
biographique ou fictionnel - mais en ce qu'elles sont homogènes aux dites traces, laissées notamment dans Les mots. Je renvoie donc à ce propos aux hypothèses avancées par le livre de Josette Pacaly et l'article de Serge Doubrovsky, qui peuvent s'articuler ensemble et se résumer en termes de fixation phallique, avec les accessoires habituels: toute-puissance et dominance anale, en l'occurrence à caractère sadomasochiste prononcé, idéalisation narcissique du même. Je mettrai simplement en relief, après eux, ce que le passage cité ci-dessus illustre parfaitement. D'une part, l'"écœurant", le "visqueux", ce qui donne "la nausée" - pour reprendre la terminologie sartrienne - ne correspond pas à l'horreur éprouvée face à la féminité mais à l'horreur du féminin en soi ${ }^{49}$; d'autre part, l'identification maternelle de l'enfant des Mots est une identification à la mère possédée par le père (le grand-père), investie sur un mode sadomasochiste ${ }^{50}$.

À qui obéirais-je? On me montre une jeune géante, on me dit que c'est ma mère. De moi-même, je la prendrais plutôt pour une sœur aînée. Cette vierge en résidence surveillée, soumise à tous, je vois bien qu'elle est là pour me servir. Je l'aime: mais comment la respecterais-je, si personne ne la respecte? [...] Les "enfants", c'est nous: pareillement mineurs et pareillement entretenus. Mais tous les égards sont pour moi. Dans ma chambre, on a mis un lit de jeune fille. La jeune fille dort seule et s'éveille chastement; je dors encore quand elle court prendre son atub" à la salle de bains; elle revient entièrement vêtue: comment serais-je né d'elle? Elle me raconte ses malheurs et je l'écoute avec compassion: plus tard je l'épouserai pour la protéger. Je le lui promets: j'étendrai ma main sur elle, je mettrai ma main sur elle, je mettrai ma jeune importance à son service. ${ }^{51}$

49 Ici encore, le Lucien de L'enfance d'un cbef ou la version sartrienne de Genet annoncent l'enfant des Mots.

50 À juste titre, Josette Pacaly analyse dans cette perspective aussi "l'identification à Grisélidis». Rappelons à sa suite le passage le plus parlant, qui s'inscrit dans le registre, déjà commenté supra, de la "suite au prochain numéron: "J'avais lu vingt fois, dans la passion, l'histoire de Grisélidis; pourtant je n'aimais pas souffrir et mes premiers désirs furent cruels: le défenseur de tant de princesses ne se gênait pas pour fesser en esprit sa petite voisine de palier. Ce qui me plaisait dans ce récit peu recommandable, c'était le sadisme de la victime et cette inflexible vertu qui finit par jeter à genoux le mari bourreau. [...] Mais je remettais chaque jour l'acquittement au lendemain; héros toujours futur, je languissais de désir pour une consécration que je repoussais sans cesse." (1964), op. cit., p. 111.

Ibid., p. 21 
Ce refus - prêté à l'enfant mais correspondant aussi bien au point de vue du narrateur - de la version oedipienne de la scène primitive, qui permet de mettre la main sur cette sœur vierge et soumise, montre un enfant actif (à la main active), conforme en somme à l'auto-présentation ultérieure de l'adulte. Ce pôle actif a toutefois son envers passif. L'enfant, "tripoté" par tous ${ }^{52}$, est au sens fort l'objet des soins de la mère ${ }^{53}$.

Quant à la visée limitée qui est la nôtre dans ce travail, cette bivalence (actif/passif) du registre de la possession/manipulation me paraît centrale. Elle permet de repérer la mise en écriture de la relation de soi à soi, en ce qu'elle repose sur l'allocation à la figure grand-paternelle d'un pouvoir d'incarnation de "Dieu le père " et de réincarnation du fils crucifié (le père mort); ce qui rend pensable la prégnance de la mort dans Les mots, et ses différents niveaux, en termes d'une sexualisation de la mort qualifiant le registre fantasmatique dans et par lequel le texte autobiographique peut accomplir sa fonction de double ou d'équivalent corporel, en deçà de la narration.

Doubrovsky relève fort bien que ce registre de la possession/ manipulation, du féminin en soi trouve là encore son expression théorique dans L'être et le néant, avec la théorisation du visqueux ${ }^{54}$. Dans ladite théorisation, le visqueux vient désigner ce qui échappe à l'appropriation sadique de l'autre, à la réduction instrumentale de l'autre à un objet manipulable ${ }^{55}$. Plus précisément même, le visqueux signifie ce qui de l'autre en tant qu'objet possédé se renverse en lieu d'une dé-possession de soi: le visqueux, c'est la possession féminine ${ }^{56}$. Traduit avec Les mots dans le registre de la relation de soi à soi, cela concerne bien évidemment "l'appropriation" de la mère en soi, c'est-à-dire l'identification à cet être féminin soumis prénommé Anne-Marie. Cette

52 Cf. ibid., p. 35.

53 Cf. la suite du passage cité ci-dessus (ibid., p. 21).

54 Serge Doubrovsky, "Sartre: retouches à un autoportrait ", op. cit.

55 Citons la définition suivante: "Il [le sadique] vise à faire prendre à l'Autre des attitudes et des positions telles que son corps paraisse sous l'aspect de l'obscène: ainsi demeure-t-il sur le plan de l'appropriation instrumentale puisqu'il fait naître la chair en agissant par la force sur l'Autre - et l'Autre devient un instrument entre ses mains - le sadique manie le corps de l'autre, pèse sur ses épaules pour l'incliner vers la terre et faire ressortir ses reins, etc. [...]" (1943, op. cit., p.453). Cf. ibid., p. 670-671. 
identification apparaît comme une soumission au "rôle" prescrit par la mère, une identification à son désir: "Anne-Marie tenait bon; elle eût aimé, je pense, que je fusse une fille pour de vrai; avec quel bonheur elle eût comblé de bienfaits sa triste enfance ressuscitée. Le Ciel ne l'ayant pas exaucée, elle s'arrangea: j'aurais le sexe des anges, indéterminé mais féminin sur les bords. " 57 Elle apparaît aussi, dans son pôle actif, comme une appropriation du rôle de lectrice 58 .

L'appropriation des mots est d'autant plus essentielle que pour l'enfant, "Platonicien par état"59, elle équivaut à l'appropriation du monde. Et s'identifier au rôle maternel signifie plus généralement s'identifier à "l'attente" des adultes, soit en termes non sartriens, à l'objet de leur désir. Pour l'enfant, lesdits adultes trouvent leur plus simple expression dans le couple œdipien formé par le grand-père et la mère. L'auto-analyste existentiel ne saurait bien évidemment nommer comme telle cette relation cedipienne père-fille car ce serait se désigner un rival tout-puissant (son grand-père) pour la possession de sa mère; alors qu'en rester au point de vue de l'enfant permet, en proclamant un "CEdipe fort incomplet "pour cause de père mort ${ }^{60}$, de se garder la mère.

\section{**}

L'auto-théorisation participe de la résistance sartrienne (en forme de roublardise) à la psychanalyse, que nous en considérions le fondement théorique (L'être et le néant) ou l'écriture autobiographique (Les mots) ${ }^{61}$. Il reste que l'enjeu premier des

57 (1964), op. cit., p. 89.

58 Cf. ibid., p. 41-43.

59 Ibid., p. 46. Ce qui vaut pour la lecture vaudra aussi bien pour l'écriture ( $c f$. ibid., p. 121, 154).

60 Cf. ibid., p. 24-25.

61 L'auto-théorisation sartrienne serait à comparer et à différencier de l'autothéorisation dans l'écriture de la cure par l'analysant. Je me permets sur ce point de renvoyer le lecteur à Jean-François Chiantaretto, "Passages à l'écrit. À propos de l'écriture de la cure par l'analysant ", Psychanalystes, $\mathrm{n}^{\circ} 38$, p. 37-54; "Écriture de l'analyse et autofiction. Le cas Serge Doubrovsky", RITM, n $\mathrm{n}^{\circ}$ 6, à paraître en décembre 1993; "L'écriture de la cure par l'analysant. Une approche psychanalytique", Psychanalyse à l'Université, à paraître en janvier 1994 . 
Mots, et c'est toute sa spécificité dans le corpus sartrien, réside dans la possibilité de se faire une scène primitive sur mesures aux mesures de la tâche autobiographique -, où il s'agit de se faire naître de ses cuvres dans la relation à l'une (la mère) et à l'autre (le grand-père incarnant le père). 